\title{
Oculocerebrofacial syndrome, Kaufman type
}

INSERM

\section{Source}

INSERM. (1999). Orphanet: an online rare disease and orphan drug data base. Oculocerebrofacial syndrome, Kaufman type. ORPHA:2707

Oculocerebrofacial syndrome, Kaufman type is characterized by psychomotor retardation, microcephaly, upslanting palpebral fissures, eye abnormalities (microcornea, strabismus, myopia, optic atrophy), high-arched palate, preauricular skin tags and micrognathia with respiratory distress. It has been described in about 10 cases. Other anomalies can be present: long thin hands and feet, ambiguous genitalia, hypertelorism, etc. An autosomal recessive mode of inheritance seems most likely. 